Florida International University FIU Digital Commons

$5-15-2012$

\title{
The Factor Structure of the Inventory of Parent and Peer Attachment in a Clinic-referred Sample of Anxious Latino Youth
}

Devi Hausman

Florida International University, devihausman@yahoo.com

DOI: $10.25148 /$ etd.FI12071105

Follow this and additional works at: https://digitalcommons.fiu.edu/etd

\section{Recommended Citation}

Hausman, Devi, "The Factor Structure of the Inventory of Parent and Peer Attachment in a Clinic-referred Sample of Anxious Latino Youth" (2012). FIU Electronic Theses and Dissertations. 652.

https://digitalcommons.fiu.edu/etd/652 


\title{
FLORIDA INTERNATIONAL UNIVERSITY
}

Miami, Florida

THE FACTOR STRUCTURE OF THE INVENTORY OF PARENT AND PEER ATTACHMENT IN A CLINIC-REFERRED SAMPLE OF ANXIOUS LATINO YOUTH

\author{
A thesis submitted in partial fulfillment of the \\ requirements for the degree of \\ MASTER OF SCIENCE \\ in \\ PSYCHOLOGY \\ by
}

Devi Hausman

2012 
To: Dean Kenneth G. Furton

College of Arts and Sciences

This thesis, written by Devi Hausman, and entitled The Factor Structure of the Inventory of Parent and Peer Attachment in a Clinic-referred Sample of Anxious Latino Youth, having been approved in respect to style and intellectual content, is referred to you for judgment.

We have read this thesis and recommend that it be approved.

Jeremy Pettit

Dionne Stephens

Wendy Silverman, Major Professor

Date of Defense: May 15, 2012

The thesis of Devi Hausman is approved.

Dean Kenneth G. Furton

College of Arts and Sciences

Dean Lakshmi N. Reddi

University Graduate School

Florida International University, 2012 


\section{DEDICATION}

I dedicate this thesis to my Ma, my family, and my boyfriend. Their encouragement, love, and support has allowed me to reach my dreams. 


\section{ACKNOWLEDGMENTS}

I would like to thank the members of my committee, Dr. Wendy Silverman, Dr. Dionne Stephens and Dr. Jeremy Pettit, for their assistance and support throughout the thesis process. I also wish to thank my major professor, Dr. Silverman for the opportunity to work successfully at the Child Anxiety and Phobia Program as well as the ladies at CAPP for their continued motivation and support. Last, a very special thank you to Dr. Silverman's post doc, Dr. Yasmin Rey for her patience, understanding, and guidance throughout my graduate career. 


\section{ABSTRACT OF THE THESIS}

THE FACTOR STRUCTURE OF THE INVENTORY OF PARENT AND PEER ATTACHMENT IN A CLINIC-REFERRED SAMPLE OF ANXIOUS LATINO YOUTH by

Devi Hausman

Florida International University, 2012

Miami, Florida

\section{Professor Wendy Silverman, Major Professor}

The present study examined the factor structure of the Inventory of Parent and Peer Attachment (IPPA; Armsden \& Greenberg, 1987) in a US sample of 282 clinicreferred anxious Latino youth (48.9\% girls; $M=10.5$ years). The IPPA is a self-report questionnaire that assesses youths' perceptions of attachment to parents and to peers and contains a three factor structure with items measuring Trust, Communication, and Alienation. Both confirmatory factor analysis and exploratory factor analysis were performed to test the factor structure of the IPPA in a sample of anxious Latino youth.

Results showed that a two factor structure best accounted for the data in the present study. These findings are inconsistent with prior research concluding that the IPPA contained a three factor structure (Armsden \& Greenberg, 1987). Results suggest that the IPPA may not capture the intended constructs among ethnically diverse clinicreferred populations. Implications of the findings are further discussed. 


\section{TABLE OF CONTENTS}

CHAPTER

PAGE

I. LITERATURE REVIEW ........................................................................... 1

Attachment Styles .................................................................................... 3

Attachment Styles and Developmental Outcomes........................................ 5

Measuring Attachment........................................................................ 7

Inventory of Parent and Peer Attachment ................................................ 9

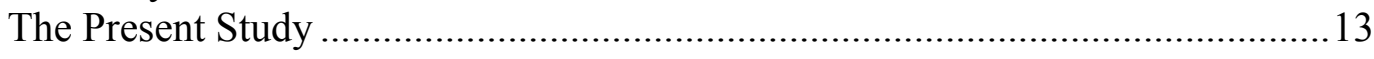

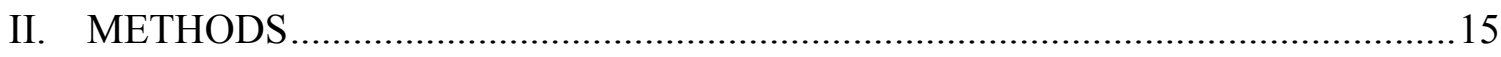

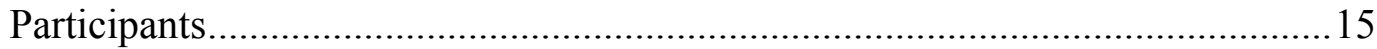

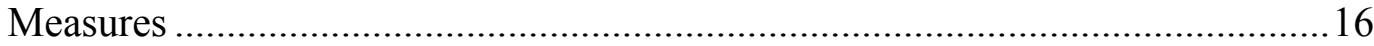

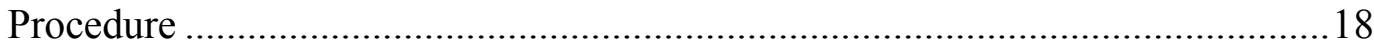

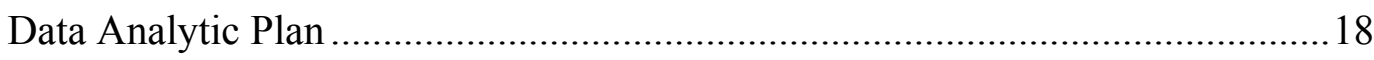

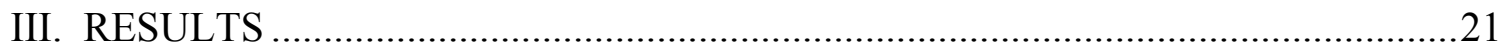

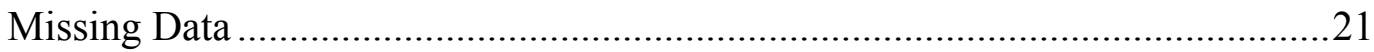

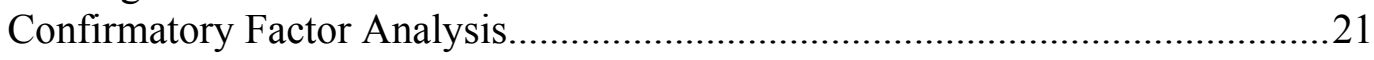

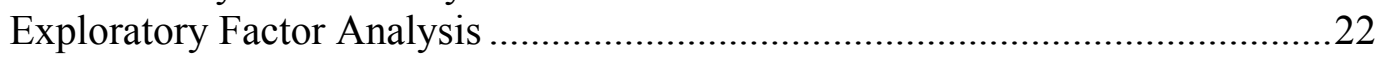

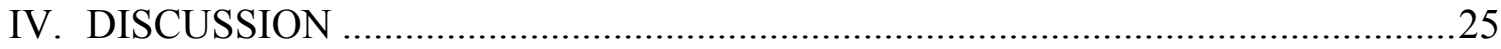

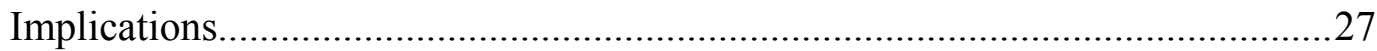

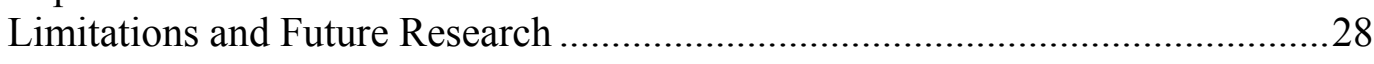

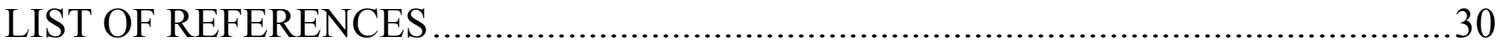

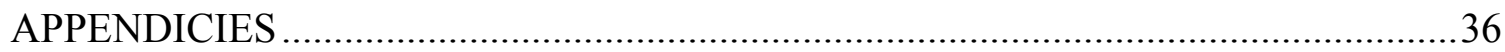




\section{CHAPTER I.}

\section{LITERATURE REVIEW}

According to theory (Bowlby 1969; 1973), attachment is a biologically centered system of behavior in which the protection and survival of the young are maintained through close proximity to caregivers. These attachment behaviors can be observed in a variety of species including primates and humans (Bowlby, 1973; Suomi, 1999). During the earliest years of their lives, infants develop an attachment bond to their caregiver. Emphasis of attachment is on the quality of attachment relationships and affectional bonds between infants and their parents or caregivers early in life. During infancy, physical proximity is the most important aspect of the infant's attachment system, whereas availability of attachment figures is most crucial during middle childhood (Bowlby, 1987). Although the formation of attachment bonds during infancy is most important to the development of secure attachment, Bowlby (1973) suggested that attachment at any age could be developed by the child's confidence in a trusted and responsive caregiver.

Bowlby (1973) hypothesized that infants develop cognitive representations, conscious and unconscious, on the basis of their early experiences with their mothers or caregivers. Influenced by Piaget's (1954) theory of cognitive development and adaptive behavior (Craik, 1943), Bowlby (1973) suggested that cognitive representations of the self and attachment figures was a natural consequence of human development

(Bretherton, Ridgeway, \& Cassidy, 1990). Coined the "internal working model," children create cognitive maps, representations, schemes, or scripts about themselves and their 
environment based on experiences and interactions with parents and significant others (Marrone, 1998).

Internal working models of the self are significant in that they demonstrate to the child how acceptable or unacceptable he or she is in the eyes of their attachment figure (Bowlby, 1973), thus forming their first concept of self esteem. For example, a child who experiences attachment figures as mainly unresponsive and dismissive is likely to develop an internal working model that mirrors these experiences (Bretherton et al., 1990; Marrone, 1998). In this example, the internal working model represents the self as undeserving or unacceptable. Research suggests that attachment beyond childhood is continually reflected in the organization of an individual's internal working model (Armsden \& Greenberg, 1987). Therefore, early attachment relationships and interactions with caregivers are imperative to the construction and maintenance of positive emotional and social development.

Research has suggested that the quality of attachment to caregivers plays a vital role in youth's behavioral and emotional responses to social and environmental challenges, including normal developmental milestones (e.g., school transition) and nonnormative life events (e.g., abuse; Muris, Meesters, \& van den Berg, 2003; Parade, Leerkes, \& Blankson, 2010). A positive attachment relationship between child and caregiver may provide a secure foundation in which the child can explore new situations and environments independently while maintaining safety and support (Ainsworth, Blehar, Waters, \& Wall, 1978). Conversely, a lack of trust or support from an unresponsive caregiver may serve as a risk factor for future developmental challenges (Papini \& Roggman, 1992). 


\section{Attachment Styles}

Mary Ainsworth illustrated her ideas on attachment relationships and attachment security by developing categories of attachment by which infants can be classified (Ainsworth et al., 1978). The development of these classifications originated from observations of infants using the Strange Situation Procedure as part of a longitudinal investigation of infant-mother attachment behaviors (Ainsworth \& Bell, 1970). The sample used in this study consisted of 56 Caucasian infants ranging from 40-51 weeks old and their mothers. The Strange Situation Procedure is a twenty-minute task that involves the observation of infant behaviors as they are introduced to a "strange situation" in which experimenters and caregivers enter and exit a room where a child is playing. During the procedure, five infant behaviors were observed as the infant's mother exited the room, during the mother's absence, and upon the mother's return. These behaviors included: 1) seeking proximity and contact; 2) maintaining contact; 3) avoiding proximity and interaction; 4) contact and interaction; and 5) searching (Ainsworth \& Bell, 1970).

On the basis of observations from the Strange Situation Procedure, Ainsworth categorized infants into three attachment categories: secure attachment, avoidant attachment, and anxious-ambivalent attachment. Avoidant attachment and anxiousambivalent attachment are considered categories of insecure attachment (Ainsworth et al., 1978; Levy, Blatt, \& Shaver, 1998). A secure attachment style is characterized by the ability of mothers to separate from their infants and then have their infants display positive emotions towards their mother upon their return. Secure infants also seek 
comfort from their mother when frightened, and use their mother as a secure base in which to explore unfamiliar situations (Ainsworth \& Bell, 1970).

Anxious-ambivalent attachment is characterized by infants exhibiting mixed or contradictory behaviors towards their mothers. These behaviors include excessive crying during separations from their mother, and are unresponsive towards their mother once they return. Avoidant attachment is characterized by infants who exhibit less distress than secure or anxious-ambivalent infants upon separation from their mother. Upon their mothers return, infants of this category display clear avoidance of interaction or close proximity to their mother. Avoidance is displayed by actions such as turning their body or head away from their mother (Ainsworth \& Bell, 1970).

Main and Solomon (1986) later recognized that infant behavior did not always resemble one of the three infant attachment styles developed by Ainsworth et al. (1978). Therefore, on the basis of several observational studies utilizing a procedure similar to the Strange Situation Procedure, Main and Solomon (1986; 1990) added a fourth category of infant attachment, namely the disorganized and/or disoriented style. The disorganized/disoriented style of infant is viewed as a type of insecure attachment quality but with distinct characteristics (Ijzendoorn, Schuengel, \& Bakermans-Kranenburg, 1999). These characteristics include displays of simultaneous contradictory behavior patterns, including freezing, confusion or apprehension (Main \& Solomon, 1986).

According to Ainsworth and Bell (1970), parenting styles and parental attributes contribute to the development of infant attachment styles. Parents of securely-attached infants are generally more available, responsive, sensitive, and autonomy-granting compared to parents of insecurely attached infants (Ainsworth \& Bell, 1970; Levy et al., 
1998). Parents of children with insecure attachments are generally unresponsive, unwilling to comfort or console, or are unavailable to their children both physically and emotionally (Collins \& Reed, 1990; Levy et al., 1998). Specifically, parents of anxiousambivalent children are often intrusive or inconsistent and parents of disorganized/disoriented children are more troubled, depressed, or abusive (Carlson, Cicchetti, Barnett, \& Braunwald, 1989; Easterbrooks, Biesecker, \& Lyons-Ruth, 2000).

\section{Attachment Styles and Developmental Outcomes}

Research on internalizing disorders in childhood and adolescence has highlighted the role of youths' attachment relationships in the development and maintenance of these disorders, including anxiety and depression (see Brumariu \& Kerns, 2010 and Colonnesi et al., 2011; Graham \& Easterbrook, 2000). For example, research has shown insecure attachment beliefs to be associated with children's depressive symptoms (e.g., Graham \& Easterbrooks, 2000; Moss et al., 2006). Moreover, attachment measures have been used to examine the link between attachment and depressive symtomology among children (e.g., Laible, 2000; Papini \& Roggman, 1992).

Attachment styles (Ainsworth et al., 1978) have been used to illustrate the connection between attachment and anxiety. For example, in a longitudinal study conducted by Warren, Huston, Egeland, \& Sroufe (1997), infants were classified as securely or insecurely attached to their caregivers. At the age of 17.5 years, children were assessed for current and past anxiety disorders using an interview schedule. Findings concluded that insecurely attached infants suffered from anxiety disorders more than their securely attached counterparts. 
In another study examining anxiety and attachment, Muris, Meesters, Merckelbach, \& HuÈlsenbeck (2000) used a non-clinical sample to assess worry in children based on attachment style. Their conclusions were consistent with Bowlby's (1973) theory that heightened levels of anxiety disorders symptomology were present in insecurely attached children compared to children who were classified as securely attached.

Infant attachment style categories have predicted several developmental outcomes. For example, children with secure attachments exhibit greater positive developmental outcomes, including higher levels of self-confidence and self worth, and greater positive emotional well-being than children with insecure attachments (Armsden \& Greenberg, 1987; Pappini \& Roggman 1992). Children with insecure attachments are more vulnerable to life stressors and display greater social and emotional difficulties, such as forming friendships and the heightened instance of conduct disorders compared to securely attached infants (Rosenstein \& Horowitz, 1996). Additionally, children with disorganized/disoriented attachments are more susceptible to stress during infancy, more aggressive in kindergarten, and more detached during young adulthood compared to infants who are classified as securely attached (Ijzendoorn et al., 1999).

Although it is evident that children with insecure attachment relationships with their caregivers may be at a disadvantage developmentally, there is some evidence that parents of children classified as insecure or disorganized also experience negative effects or undesirable outcomes (Minde, Minde \& Vogel, 2006). For example, insecure motherchild attachment patterns have been associated with maternal depression and anxiety (Carlson et al., 1989; Minde et al., 2006; Easterbrooks, Biesecker, \& Lyons-Ruth, 2000). 
Additionally, parents with insecure attachment relationships are more troubled or abusive (Belsky \& Cassidy, 1994).

\section{Measuring Attachment}

There are a number of methods used to measure attachment relationships and behaviors in youth, including observational or separation-reunion methods (i.e., Strange Situation Procedure; Ainsworth et al., 1978), interviews (e.g., the Child Attachment Interview; CAI; Shmueli-Goetz, Target, Fonagy, \& Datta, 2008) and self-report questionnaires (e.g., Attachment Questionnaire for Children; AQC; Muris, Mayer, \& Meesters, 2000; Security Scale; Kerns, Aspelmeier, Gentzler, \& Grabill, 2001) and the Inventory of Parent and Peer Attachment; IPPA; Armsden \& Greenberg, 1987).

Separation-reunion methods include the Strange Situation Procedure (described

earlier). The separation-reunion method for measuring attachment is commonly used with infants (e.g., Bates, Maslin, \& Frankel, 1985; Lewis, Feiring, McGuffog, \& Jaskir, 1984). Studies using observational methods have yielded excellent test-retest and inter-rater reliability estimates (coefficients $=.98$ to $.99 ; .93$ to.97, respectively). However, authors did not report specific findings related to validity. Limitations of this procedure includes the lack of consideration for individual differences in the observed patterns of behavior, is inappropriate for all ages, and assumes brief separations and reunions have the same meaning for all children across cultures (Ainsworth \& Bell, 1970; Rutter, 1995).

Another method for measuring attachment is using interviews. One widely used interview to measure attachment in youth is the Child Attachment Interview (CAI; Shmueli-Goetz et al., 2008). The CAI is a downward extension of the Adult Attachment Interview (AAI; Main \& Goldwyn, 1984) for use with children ages 8 to 13 years old 
(Shmueli-Goetz et al., 2008). Specifically, the CAI measures the degree in which children perceive their caregiver to be accessible and available. The development of this measure was based on Bowlby's (1987) theory, which posits that during middle childhood, the availability of their attachment figure is more important than physical proximity.

The CAI has been validated using both a clinical and non-clinical sample, uses developmentally appropriate wording, and analyzes narrative interviews relying on both verbal (what the child says) and nonverbal (the child's body language) communication. Although still young in its development, the CAI yielded a good inter-rater reliability estimate $(\kappa=.88)$. Convergent validity has been established using measures of separation anxiety and middle childhood, although the coefficient of agreement was only modest (Shmueli-Goetz et al., 2008). Additional research is needed to further demonstrate convergent validity as well as develop accurate coding methods (Shmueli-Goetz et al., 2008).

Self-report questionnaires are of the most common methods for collecting data with children and adolescents to assess attachment (e.g., Brumariu \& Kerns, 2010; Hersen 1998). Questionnaires measure individual's perceptions of attachment. Advantages of this method include cost-effectiveness, are easy to administer, and are less time consuming than other methods used to assess attachment. They are also important in the research process, as data are analyzed to test predictive hypotheses. The most widely used self-report questionnaires measuring attachment beliefs include the Security Scale (Kerns, Aspelmeier, Gentzler, \& Grabill, 2001), the Attachment Questionnaire for Children (AQC; Muris, Mayer, \& Messters, 2000), and the Inventory of Parent and Peer Attachment (IPPA; Armsden \& Greenberg, 1987). 
The Security Scale (Kerns et al., 2001) is a 15-item measure that assesses youth's perceptions of attachment relationships during middle childhood and early adolescents. The Security Scale presents children with descriptions of two types of children and asks which type of child they are most like. Items are scored from 1 to 4 , with greater attachment security represented by a higher score. Scores on the Security Scale showed adequate internal consistency (Cronbach's alpha $=.93$ ) as well as some evidence of divergent and convergent validity based on security scores correlated with self esteem, peer acceptance, and behavioral conduct, physical appearance and scholastic competence (Kerns, et. al., 1996).

Adapted from Hazan and Shaver's (1987) measure assessing attachment patterns in adults, the AQC (Muris et al., 2000) is a 1-item questionnaire that relies on children's report of their perceptions of attachment in close relationships. Similar to the Security Scale, children are provided three descriptions of perceptions about relationship with their peers and asked to choose which description is most like themselves. Items are related to one of three attachment style patterns consisting of secure attachment, avoidant attachment, or ambivalent attachment. Because the AQC is a 1-item measure, internal consistency coefficients have not been computed. In addition, test-retest reliability estimates have not been reported (Muris et al., 2001).

\section{Inventory of Parent and Peer Attachment}

Of interest in the present study is the IPPA (Armsden \& Greenberg, 1987), which is a 53 item self-report questionnaire that assesses youths' perceptions of attachment to parents and to peers. The original IPPA was comprised of two scales: The Parent scale, which consists of 28 items and the Peer scale, which consists of 25 items. The IPPA was 
later revised (Armsden \& Greenberg, 1989). The Parent scale was divided into a Mother scale and Father scale each containing 25 identical items. Three items on the Peer scale were omitted to make all three scales uniform.

Items on the IPPA scales (i.e., Mother, Father, and Peer) are related to behavioral and cognitive/affectional dimensions of attachment. Based on Bowlby's theory of attachment (Bowlby, 1969; 1973), the IPPA was designed to measure specifically the degree of trust in the accessibility and responsiveness of youths' attachment figures and peers and the degree of anger and/or hopelessness that result from unresponsive or inconsistently unresponsive attachment figures and peers (Armsden \& Greenberg, 1987).

The original article that evaluated the IPPA consisted of 179 college students, ages 16 to 20 years old. Factor analyses conducted on the items of the IPPA yielded a three factor structure for the Parent and Peer Scales, respectively: Trust, Communication, and Alienation. Overall quality of attachment to parents and peers, respectively, was defined as the degree of Trust and Communication relative to Alienation (Armsden \& Greenberg, 1987).

The IPPA's three subscales revealed good internal consistency (alpha) coefficients (.86 to .91$)$. No internal consistency was reported for the total score. Testretest reliability over a three week period was examined and the total score yielded an average reliability (coefficient $=.93$ ). Furthermore, convergent validity was supported using Pearson's $r$ correlations with additional measures of family conflict, cohesion, and support (Armsden \& Greenberg, 1987).

The IPPA has been used in a number of studies conducted to examine the association between attachment beliefs and internalizing disorder symptomology (e.g., 
Burge et al., 1997; Muris et al., 2001). Furthermore, the subscale scores of the IPPA are used in these studies to predict outcomes related to attachment beliefs. Specifically, subscale scores are used to classify attachment styles among children and adolescents (Armsden \& Greenberg, 1987).

In their study, Armsden and Greenberg (1987) demonstrated how the subscales scores of the IPPA could be used to classify individuals on the basis of their attachment levels. The authors examined attachment security based on subscale scores by placing individuals in high security, medium security, and low security groups. Individuals scoring in the medium range were excluded from analysis to theoretically define two distinct attachment comparison groups, individuals with high attachment security and individuals with low attachment security.

Although the IPPA has been widely used in the clinical and developmental child psychology literature (e.g., Abela et al., 2005; Costa \& Weems, 2005; Parade et al., 2010), little research has been conducted to evaluate the IPPA's three factor structure. Pace, San Martini, and Zavattini (2011) is one of the few studies to evaluate the factor structure of the IPPA. Using a community sample of 1059 Italian middle and high school students, Pace, San Martini, \& Zavattini (2011) tested the three factor model of the IPPA (Trust, Communication, and Alienation) as well as a two factor (Trust-communication and Alienation) and a single factor model (attachment security). Exploratory factor analyses and confirmatory factor analysis revealed the three factor model had the best fit, although all three dimensions (trust, communication, and alienation) were strongly interrelated (Pace, San Martini, \& Zavattini, 2011). 
Similarly, Guarnieri, Ponti, and Franca (2010) examined the factor structure of the IPPA using a community sample of 1183 adolescents residing in central Italy. Confirmatory factor analyses supported the three factor structure (Trust, Communication, and Alienation) proposed by Armsden and Greenberg (1987) among this sample of Italian youth.

In another study testing the factor structure of the IPPA, Johnson, Ketring, and Abshire (2003) used a sample of 212 parents and 89 adolescents drawn from a family therapy program in the Midwest. Using both confirmatory factor analysis and exploratory factor analysis, results showed that the revised version of the IPPA yielded a two factor structure, trust/avoidance and communication. The sample population consisted of individuals referred to therapy based on reports of abuse or neglect, as well delinquent behaviors among adolescence. The authors posit that the low-income, less educated population used in their study contributed to the variation in findings compared to the predominantly Caucasian, collegiate sample used by Armsden and Greenberg (1987).

Accounting for $16 \%$ of the population, Latinos are the fastest growing and largest minority group in the United States (US Census Bureau, 2006). Latino youth also present with high rates of anxiety disorders (Ginsburg \& Silverman, 1995) and have reported higher rates of depression compared to non-Hispanic Blacks and non-Hispanic Whites (Center for Disease Control and Prevention, 2006). Despite these findings, there is a lack of research devoted specifically to attachment or internalizing disorder measurement within the Latino community (Carter, Mitchell, \& Sbrocco, 2012). This is particularly problematic because research has suggested that minority populations may conceptualize various constructs such attachment, anxiety and depression differently than European 
Americans (e.g., Anderson \& Mayes, 2009; Carter et al., 2012; Crockett, Randall, Shen, Russell, \& Driscoll, 2005; Minde et al., 2006).

Although the IPPA total scale and subscales have been widely studied in relation to attachment and childhood internalizing disorders, (e.g., Burge et al., 1997; Costa \& Weems, 2005; Papini \& Roggman, 1992) its three factor structure has not been examined among minority populations, making it is unclear whether the three factor structure is applicable in Latino youth. Differences or similarities in the quality of attachment as it relates to anxiety or other outcomes may be due to measurement problems if the three factor structure of the IPPA cannot be replicated in samples of non-Caucasian youth (Sue, 1999).

\section{The Present Study}

The purpose of the present study was to examine the three factor structure of the IPPA Mother scale in a sample of anxious Latino youth, ages 7 to 16 years old. Confirmatory factor analysis was used on MPLUS to test a single factor model and a correlated factor model. Additionally, exploratory factor analysis was performed in SPSS to test the factor structure of the IPPA Mother scale in a clinic sample of anxious Latino youth.

Because there is a lack of prior research, specific hypotheses regarding the behavior of factors of the IPPA in a clinic sample of Latino youth were not formulated. As such, all analyses performed in the present study were considered exploratory. The research questions investigated in the present study asked whether the three factor structure of the IPPA Mother scale as proposed by Armsden and Greenberg (1989) would hold for a clinic sample of anxious Latino youth. Additionally, an alternate measurement 
model was sought that may better account for the data in a clinic sample of anxious Latino youth. 


\section{CHAPTER II.}

\section{METHODOLOGY}

\section{Participants}

Participants consisted of 282 Latino youth (ages 7-16 years old, $M=10.5$; $S D=2.33$ ) drawn from the Child Anxiety and Phobia Program's (CAPP) archival data collected over five years (2003-2008). The sample consisted of 138 girls and 144 boys $(48.9 \%, 51.1 \%$, respectively). All participants identify themselves as Latino. Most participants $(70.6 \%)$ were born in the US, $5.0 \%$ were born in Cuba, $2.2 \%$ were born in other Caribbean countries, $10.5 \%$ were born in Central or South American countries, one participant (.4\%) was born in the Middle East, and $11.3 \%$ did not report their country of origin. Of the $70.6 \%$ of participants born in the US, $29.4 \%$ of participant mothers were born in Cuba. In terms of annual income, $40.8 \%$ of families earned less than $\$ 40,999$, $18.4 \%$ earned $\$ 41,000$ to $\$ 60,999,11.7 \%$ earned $\$ 61,000$ to $\$ 80,999$, and $16.3 \%$ earned $\$ 81,000$ to $\$ 150,000+$. Thirty-five families $(12.4 \%)$ did not report annual income.

Primary referrals to CAPP included school counselors, mental health professionals, and pediatricians. On the basis of an initial telephone screen for eligibility, participants were evaluated for admittance to CAPP if mothers reported their children as having excessive fear or anxiety-related symptoms. Youth participants were included if the parent reported them to have difficulties related to anxiety symptoms during an initial telephone screen. Exclusionary criteria for participation included developmental delays (i.e., autism) and severe psychopathology (i.e., schizophrenia).

Of the 254 youth for whom diagnoses were obtained, $83.2 \%$ of youth met DSMIV diagnostic criteria for an anxiety disorder, anywhere in their diagnostic criteria, with 
the majority of youth $(80.5 \%)$ meeting criteria for a primary anxiety disorder based on the Anxiety Diagnostic Interview Schedule for DSM-IV: Child and Parent Versions (ADIS-IV: C/P; Silverman \& Albano, 1996). Of the remaining 55 youth that did not meet criteria for an anxiety disorder anywhere in their diagnostic profile, 27 met criteria for other disorders and 13 did not meet criteria for any disorder. Fifteen youth cases did not have diagnosis information available.

Table 1 presents the primary diagnoses for the sample of youth. As shown on Table 1, the most common primary diagnoses in this sample of youth were separation anxiety disorder (34.4\%), followed by social phobia (19.5\%), specific phobia (11.3\%), and generalized anxiety disorder (10.6\%). The majority of participants $(71.7 \%)$ had at least one comorbid disorder. Although a minority of youth participants did not meet diagnostic criteria for any anxiety disorder, all participants completed initial questionnaires, which included the Inventory of Parent and Peer Attachment (IPPA; Armsden \& Greenberg, 1989).

\section{Measures}

Demographics. Mothers of youth completed an information sheet comprised of questions regarding the demographics of both the parent and child, including ethnicity and race, child's age and gender, and socio-economic status.

Anxiety Disorders Interview Schedule for DSM-IV: Child and Parent Versions

(ADIS-IV: C/P; Silverman \& Albano, 1996). The ADIS-IV: C/P is a semi-structured diagnostic interview that contains questions created to assess the presence of major disorders (e.g., attention deficit hyperactivity disorder, conduct disorder) as well as anxiety disorders (e.g., separation anxiety, generalized anxiety disorder) in youth based 
upon DSM-IV criteria (American Psychiatric Association, 1994). Diagnoses revealed by the ADIS-IV: C/P is based on both separate youth and parent reports and composite diagnoses based on combined youth and parent reports.

Inventory of Parent and Peer Attachment (IPPA; Armsden \& Greenberg, 1987) As previously stated, the present study used the revised version of the IPPA (Armsden \& Greenberg, 1989). The original IPPA was comprised of a Parent and Peer Scale. The revised IPPA separated the Parent Scale into Mother and Father scales (IPPA-M and IPPA-F, respectively) containing identical items for each scale. For the present study, the Mother scale was used.

The IPPA-M consists of 25 items that assess youth's perceptions of attachment to their mothers. Example of items on the IPPA-M include, "my mother respects my feelings" and, "my mother expects too much from me.” Youth rate each item on a 5-point Likert scale (Never/almost never true, not very often true, sometimes true, often true, and almost always/always true. The IPPA-M contains three subscales: Trust, Communication, and Alienation. Total attachment is calculated by measuring the degree of Trust and Communication relative to Alienation.

Internal consistency (alpha) coefficients for the IPPA's three subscales ranged from .86 to .91 . In the present study, alphas for the subscales ranged from .67 to .77 . A test-retest reliability estimate over a three-week period for the total score was .93 (Armsden \& Greenberg, 1987). Furthermore, the IPPA has evidence for convergent validity with measures of family conflict, cohesion, and support, with attachment scores most highly correlated with the measures of social self concept $(r=.57, p<.001)$ (Armsden \& Greenberg, 1987). 


\section{Procedure}

After an initial telephone screen for eligibility, parents and youth provided informed consent/assent upon arrival at CAPP. Youth and parents were then administered an assessment battery that consisted of the ADIS-IV:C/P and a number of questionnaires, including the IPPA-M. The IPPA-M and other questionnaires were administered by trained advanced undergraduate or graduate research assistants. Directions for each questionnaire as well as individual items of questionnaires were read aloud to younger participants or participants with reading difficulties.

\section{Data Analytic Plan}

Confirmatory factor analyses (CFAs) were conducted on the items of the IPPA-M to test the fit of two different factor models: 1) a single factor model; and 2) a correlated three factor model. Examination of univariate indices of skewness and kurtosis for items on the IPPA-M revealed four items with skewness in the data and five values of kurtosis that were greater than an absolute value of $2.0(1.996,2.652,3.238 .2 .552$ and 3.453, $7.288,10.262,6.047,4.400$, respectively). Because of the significant kurtosis present in the data, the fit of the different factor models was evaluated using an estimator robust to non-normality using the Huber White algorithm (MLR) in MPlus 6.1 (Muthen \& Muthen, 2007).

The two factor models were statistically compared using the Satorra-Bentler ChiSquared Difference Test (Muthen \& Muthen, 2007; Satorra \& Bentler). To examine the fit of the factor models, a range of global fit indices were used, which included indices of absolute fit, relative fit, and fit with a penalty function for lack of parsimony (Bollen \& Long, 1993). The chi-square test of model fit was used as an index of absolute fit. This 
index should be statistically nonsignificant. However, obtaining a nonsignificant chisquare is not likely with large sample sizes (e.g., Kline, 1998; Marsh, Balla, \& McDonald, 1988). Thus, other indices were included that are less dependent on sample size than the chi-square test. These indices include the Comparative Fit Index (CFI), the Tucker-Lewis Index (TLI), and the Root Mean Square Error of Approximation (RMSEA). The CFI and TLI are indices of relative fit that range from 0 to 1 . Factor models that yield CFI and TLI values close to .95 or greater are considered to be a good fit to the data (Hu \& Bentler, 1999). The RMSEA is an index with a penalty function for lack of parsimony. Factor models yielding RMSEA values of .05 or less are considered to be a good fit to the data (Browne \& Cudeck 1993).

In addition to the global fit indices, more focused tests of fit were pursued. These included examination of Heywood cases and modification indices (MIs). Heywood cases (or offending estimates) are parameter estimates that have out of range values, such as factor correlations that exceed 1.00, negative factor variances, or negative error variances (Brown, 2006). Modification indices indicate the presence of fixed or constrained parameters in a model that, if freely estimated, would improve the fit of the model. Specifically, MIs reflect an approximation of how much the overall chi-square of model fit would be reduced if such parameters were freely estimated. The value of the MI is viewed as the difference in the chi-square between two models, where in one model the parameter is fixed and in the other model the parameter is freely estimated. Because MIs are viewed as a chi-square difference with a single degree of freedom, MIs of 3.84 or greater suggests that the overall fit of the model could be significantly improved at $p<$ 
.05 if the parameter was freely estimated. However, this value is typically rounded to 4.00 (Jaccard \& Wan, 1996).

The two factor models were statistically compared against each other using the traditional nested chi-square test. This involved taking the difference between the chisquare values and the difference in the degrees of freedom for each model being tested. A statistically significant chi-square difference indicates that the less restrictive factor model (i.e., three factor model) fits statistically significantly better than the more restrictive factor model (i.e., unidimensional factor model). 


\section{Chapter III.}

\section{RESULTS}

\section{Missing Data}

Eleven percent of youth cases had at least one missing value on a single item. Missing data were examined to determine whether there was systematic bias in the patterning of missing data. Missing data bias was assessed by computing a dummy variable reflecting the presence or absence of missing data for each indicator in the CFA and then this dummy variable was correlated with all other indicators as well as an array of demographic variables. No meaningful or significant bias was observed in any instance. Given the absence of systemic bias in the patterning of missing data, Full Information Maximum Likelihood (FIML) was used in the present study as the missing data estimation approach (Wothke, 2000).

\section{Confirmatory Factor Analyses}

The first model tested was a single factor model. The chi-square index for the single factor model was $722.620(\mathrm{df}=275, p<.001)$, the CFI was .74, the TLI was .71 and the RMSEA was .08. Thus, all fit indices indicated that the single factor model yielded a poor fit to the data of Latino youth in the present study.

The second model tested was a correlated factor model comprised of three factors: Trust, Communication, and Alienation. The chi-square index was $106.682(\mathrm{df}=272, p$ $<.001$ ), the CFI was .83 , the TLI was .81, and the RMSEA was .076. With the exception of the RMSEA, fit indices indicated that the three factor model yielded a poor fit to the data. 
Comparisons of the single factor and three factor models revealed that the correlated three factor model fit statistically significantly better than the single factor model $\left(\chi^{2}\right.$ diff $\left.[3]=106.682, p<.001\right)$. However, as noted, the three factor model generally yielded a poor fit to the data.

Given that the CFA indicated the three factor structure originally found by Armsden and Greenberg (1987) did not provide a good fit, exploratory factor analyses (EFA) were conducted to determine the factor structure of the IPPA-M in this sample of Latino anxious youth.

\section{Exploratory Factor Analyses}

EFAs with a direct oblimin rotation were performed using principle-axis factoring (PAF) on SPSS. Principal axis factoring was used because it is recommended when there are violations of normality in the data (Fabrigar, Wegener, MacCallum, \& Strahan, 1999), as is the case with the data in the present study. Oblimin rotation is an oblique rotation that allows factors to be correlated, and is generally recommended over orthogonal methods of rotation that assume the factors are uncorrelated, which is a rarity in the social sciences (Costello \& Osborne, 2005).

To determine the optimal number of extracted factors in the EFAs, the scree test was used. The scree test involves examining the graph of the eigenvalues and looking for the natural break point in the data where the curve flattens out. The number of data points above the break (not including the point at which the break occurs) is usually the number of factors to retain (Catell, 1966).

Figure 1 illustrates the scree plot generated by the EFA in the sample. The scree plot suggests the presence of 2 or 3 factors inconsistent with the original three factor 
structure previously found by Armsden and Greenberg (1987). In cases where the number of factors suggested by the scree test differs from the predicted number of factors on the basis of previous research, it has been recommended that multiple EFAs are run with the numbers of factors to retain set manually in each run - once at the predicted number of factors, again at the number of factors suggested by the scree test, and then at numbers above and below those numbers (Costello \& Osborne, 2005). After rotation, the item loading tables are compared and the factor solution that best fits the data is one in which there are item loadings above .32 (as recommended by Tabachnick \& Fidell, 2001), no or few items cross loading (an item that loads at .32 on two or more factors), and no factors with fewer than three items (Costello \& Osborne, 2005).

Therefore, the data in the present study were run three times with each run setting the number of factors extracted at two, three, and four. Because previously conducted CFAs revealed that the single factor model did not provide a good fit to the data, factor analyses were not conducted setting the number of factors extracted at one.

The EFA was run with the number of factors set to two. Examination of the pattern matrix revealed that all of the factor loadings of the items on the IPPA-M were above .32 , with the exception of item 3 . Item 3 did not load on either factor. There were no items cross loading, and none of the factors had fewer than three items, as 15 items loaded on factor 1 and 9 items loaded on factor 2 . The two factor model accounted to $43.14 \%$ of the variance among the items.

The second EFA was run with the number of factors set to three. Examination of the pattern matrix revealed that all the factor loadings of the items on the IPPA-M were above .32 , except for item 3 . Again, item 3 did not load on any of the 3 factors. Items 2 
and 22 cross loaded onto the first and third factors. The third factor was comprised of only these two items, 2 and 22 . The three factor model accounted for $47.97 \%$ of variance among the items.

The third EFA was run with the number of factors set to four. Examination of the pattern matrix revealed that all the loadings of the items on the IPPA-M were above .32 . However, there were several items cross loading onto two factors. Specifically, item 19 cross loaded onto factors 1 and 4; items 1,2, and 22 cross loaded onto factors 1 and 3; items 10,11 , and 17 cross loaded onto factors 2 and 3; and item 7 cross-loaded onto factors 3 and 4 . The four factor model accounted for $52.43 \%$ of variance among items.

Examination of the scree plot and pattern matrices suggested that two factors best explained the present study's data (see Table 2). The first factor accounted for $31.76 \%$ of total variance in the items and contained 15 items that suggested respect, understanding, and trust. The second factor accounted for $11.38 \%$ of total variance in the items and contained 9 items that suggested apathy, emotional distancing, and withdrawal. The correlation between the two factors was modest $(r=-.30)$, suggesting that the factors were related but distinct factors. 


\section{CHAPTER 1V.}

\section{DISCUSSION}

The purpose of the present study was to evaluate the three factor structure of the Mother scale of the Inventory of Parent and Peer Attachment (IPPA; Armsden \& Greenberg, 1987) in a clinic-referred sample of anxious Latino youth. Although the three factor model fit statistically significantly better than a single factor model representing a unidimensional construct of attachment, the three factor model did not provide an adequate fit to the data. Thus, results were inconsistent with Armsden and Greenberg's (1987) findings. In light of this, it was necessary to conduct EFAs to determine the factor structure of the IPPA-M in this clinic sample of anxious Latino youth.

The present study extends prior research related to the factor structure of the IPPA. As previously outlined, inconsistencies in research findings regarding the IPPA's three factor structure have been reported among Caucasian and minority populations (e.g., Pace et al., 2011; Johnson et al., 2003). The results obtained in the present study highlight previous conclusions that the IPPA's three factor structure is not supported when examined among minority or clinical populations (Johnson et al., 2003). Although the analyses conducted are preliminary in nature and examine one sample of youth, the present study provides the groundwork for which further conclusions may be drawn using samples of other minority or non-normal populations.

Results of EFAs revealed that a two factor model best explained the present study's data. The first factor was comprised of items that suggested themes consistent with secure attachment beliefs. Specifically, items of this factor were related to feelings of respect, trust, and understanding towards mothers (e.g., "My mother helps me to 
understand myself better," and "my mother respects my feelings." The second factor had items related to insecure attachment beliefs. Items of this factor suggested feelings of apathy and emotional distancing towards mothers (e.g., "My mother doesn't understand what I'm going through these days," and "I get upset a lot more than my mother knows about."

These findings reveal that the Trust and Communication factors of the IPPA originally reported by Armsden and Greenberg (1987) are not distinct factors in this sample of anxious Latino youth. Thus, youth in the present study may conceptualize trust and communication as one coherent construct. These findings are consistent with prior research has suggesting that minority populations may conceptualize various constructs such as attachment and anxiety differently than European Americans (e.g., Anderson \& Mayes, 2009; Carter et al., 2012, DeMinzi, 2006; Minde et al., 2006).

Item 3 ("I wish I had a different mother") of the IPPA-M, did not load onto any factor during data analysis. Originally, Armsden and Greenberg reported this item as a reverse scored item that loaded onto the Trust factor. Research shows that the Latino culture emphasizes the importance of interdependence, support, and family ties more so than the mainstream U.S. culture (e.g., Bulcroft, Carmody, Bulcroft, 1996; Driscoll et al., 2008). With that in mind, this is the only item of the IPPA-M that suggests the replacement of the youth's mother, a notion that may not be congruent with Latino cultural beliefs. Further research is warranted to obtain additional explanations for this items lack of conformity to a single factor. 


\section{Implications}

The factor structure of the IPPA-M was analyzed by Armsden and Greenberg (1987) using of a sample of predominantly Caucasian adolescents. The inconsistent findings related to the factor structure in a clinic sample of anxious Latino youth suggests that this measure may not yield valid results among ethnically diverse cultures. As

previously mentioned, results showed that this sample of Latino youth may conceptualize attachment differently than European Americans. Administering measures that are culturally sensitive can result in undesirable outcomes. For example, measures assessing attachment that were developed for one population (i.e. European Americans) that are used in another population (i.e. Latinos) can distort prevalence estimates and incorrectly report ethnic disparities in mental health, which can lead to erroneous policy initiatives (Crockett et al., 2005).

The subscale scores of the IPPA have been used in previous studies to examine attachment and child symptomology (e.g., Burge et al., 1997; Muris et al., 2001, Papini \& Roggman, 1991). Misclassifications or inaccurate results can occur if standard criterion is used for a minority population in which measures were not designed to evaluate (Crockett et al., 2005). For example, in Armsden and Greenberg's study (1987), youth were associated with high, medium, or low security levels based on subscale scores. The standard cutoffs that were created to classify youth with high or low security may not have been appropriate for use with minorities if attachment is conceptualized differently compared to European Americans. 


\section{Limitations and Future Research}

In the present study, there are some limitations that warrant attention when interpreting results. First, the present study used a sample of Latino youth presented to an anxiety disorders specialty clinic. The pattern of findings using samples of youth who have other types of psychiatric disorders is unclear. Therefore, future studies should incorporate clinical populations from additional sources.

Second, although an entirely Latino youth sample was used, multiple subgroups within the Latin countries exist with distinct cultures and acculturation levels (U.S. Department of Health and Human Services, 2001). The number of youth in each subgroup (e.g., Cuban, Venezuelan) was too small to conduct statistical comparisons. Future research may benefit by extending this study to examine variation among different subgroups within the Latin community.

Third, the present study used exploratory factor analysis to interpret the data. Therefore, the nature and design of the results obtained is simply exploratory. Caution is warranted in drawing substantive conclusions based on EFA results as it is not designed to test hypotheses or theories (Costello \& Osborne, 2005). Therefore, it is important for future research to conduct CFAs to examine the two factor structure found with this sample using an independent sample of anxious Latino youth.

Lastly, this study relies solely of youth's self report of perceptions of attachment to their mothers. Although self-report methods are the more favorable method for assessing youth attachment (Achenbach et al., 1987), confounds of the self-report method such as social desirability may still exist. Therefore, it would be beneficial for future 
research to incorporate a multi-method or multi-informant approach in conjunction with self-report questionnaires to assess attachment beliefs. 


\section{LIST OF REFERENCES}

Abela, R., Hankin, B., Haight, E., Adams, P., Vinokuroff, T., \& Trayhern, L. (2005). Interpersonal vulnerability to depression in high-risk children: The roe of insecure attachment and reassurance seeking. Journal of Clinical and Adolescent Psychology, 34(1), 182-192. doi:10.1207/s15374424jccp3401_17

Achenbach, T.M., McConaughy, S.H., \& Howell, C.T. (1987). Child/adolescent behavioral and emotional problems: Implications of cross-informant correlations for situational specificity. Psychological Bulletin, 101, 213-232. doi:10.1037/0033-2909.101.2.213

Ainsworth, M., \& Bell, S. (1970). Attachment, exploration, and separation: Illustrated by the behavior of one-year-olds in a strange situation. Child Development, 41, 4967. Retrieved from http://www.jstor.org/stable/1127388

Ainsworth, M., Blehar, M., Waters, E., \& Wall, S. (1978). Patterns of attachment: A psychological study of the strange situation. Hillsdale, NJ: Lawrence Erlbaum.

Armsden, G.C., \& Greenberg, M.T. (1987). The inventory of parent and peer attachment: Individual differences and their relationship to psychological well-being in adolescents. Journal of Youth and Adolescents, 16(5), 427-452. doi:10.1007/BF02202939

Armsden, G.C., \& Greenberg, M.T. (1989). The inventory of parent and peer attachment (IPPA). Unpublished report. WA, USA: University of Washington.

Bates, J.E., Maslin, C.A. \& Frankel, K.A. (1985). Attachment security, mother-child interaction, and temperament as predictors of behavior-problem ratings as age three years. Monographs of the Society for Research in Child Development, 50(1/2), 167-193. Retrieved from http://www.jstor.org/stable/3333832

Bollen, K.A. (Ed). \& Long, S.J. (Ed). (1993). Testing structure equation models. Thousand Oaks, CA: Sage Publications.

Bowlby, J. (1969). Attachment and loss: Vol. 1. Attachment. New York: Basic Books.

Bowlby, J. (1973) Attachment and loss: Vol. 2. Separation: Anxiety and anger. New York: Basic Books.

Bretherton, I., Ridgeway, D., \& Cassidy, J. (1990) Assessing internal working models of the attachment relationship: An attachment story completion task for 3-year olds. In M. T. Greenberg, D. Cicchetti, \& E.M. Cummings (Eds.), Attachment in the preschool years: Theory, research, and intervention (pp. 273-294). Chicago, IL: University of Chicago Press. 
Browne, M. W., \& Cudeck, R. (1993). Alternate ways of assessing model fit. In K. A. Bollen \& J. S. Long (Eds.), Testing structural equation models (pp. 136-162). Newbury Park, CA: Sage.

Brumariu, L.E. \& Kerns, K.A. (2010). Parent-child attachment and internalizing symptoms in childhood and adolescents: A review of empirical findings and future directions. Development and Psychopathology, 22, 177-203. doi:10.1016/j.appdev.2008.06.002

Bulcroft, R. A., Carmody, D. C., \& Bulcroft, K. A. (1996). Patterns of parental independence giving to adolescents: Variations by race, age, and gender of child. Journal of Marriage \& the Family, 58, 866-883. Retrieved from http://www.jstor.org/stable/353976

Burge, D., Hammen, C., Davila, J., Daley, S.E., Paley, B., Lindberg, N., \& Rudolph, K.D. (1997). The relationship between attachment cognitions and psychological adjustment in late adolescent women. Development and Psychopathology, 9, 151167. doi:10.1017/S0954579497001119

Carlson, V., Cincchetti, D., Barnett, D., \& Braunwald (1989). Disorganized/disoriented attachment relationships in maltreated infants. Developmental Psychology, 25(4), 525-531. doi:10.1037/0012-1649.25.4.525

Carter, M.M., Mitchell, F.E., \& Sbrocco, T. (in press). Treating ethnic minority adults with anxiety disorders: Current status and future recommendations. Journal of Anxiety Disorders (2012), doi:10.1016/j.janxdis.2012.02.002

Center for Disease Control and Prevention. (2006). Depression Statistics. Retrieved from http://www.cdc.gov/mentalhealth/data_stats/depression.htm

Colonnesi, C., Draijer, E.M., Stams, J.M., Van der Bruggen, C.O., Bogels, S.M., \& Noom, M.J. (2011). The relation between insecure attachment and child anxiety: A meta-analytic review. Journal of Clinical Child \& Adolescent Psychology, 40(4), 630-645. doi:10.1080/15374416.2011.581623

Costa, N.M. \& Weems, C.F. (2005). Maternal and child anxiety: Do attachment beliefs or children's perceptions of maternal control mediate their association? Journal of Social Development, 14(4), 574-590. doi:10.1111/j.1467-9507.2005.00318.x

Craik, K. (1943). The Nature of Explanation. New York: Cambridge University Press

Crockett, L., Randall, B., Shen, Y., Russell, S., \& Driscoll, A. (2005). Measurement equivalence of the Center for Epidemiological Studies depression scale for Latino and Anglo American adolescents: A national study. Journal of Consulting and Clinical Psychology, 73(1), 47-58. doi:10.1037/0022-006X.73.1.47 
DeMinzi, M.C. (2006). Loneliness and depression in middle and late childhood: The relationship to attachment and parental styles. Journal of Genetic Psychology, 167(2), 189-210. doi:10.3200/GNTP.167.2.189-210

Driscoll, A. K., Russell, S. T., \& Crockett, L. J. (2008). Parenting styles and youth wellbeing across immigrant generations. Journal of Family Issues, 29, 185-209. doi:10.1177/0192513X07307843

Easterbrooks, M.A., Biesecker, G., \& Lyons-Ruth, K. (2000). Infancy predictors of emotional availability in middle childhood: The roles of attachment security and maternal depressive symptomology. Attachment \& Human Development, 2, 170178. doi:10.1080/14616730050085545

Fabrigar, L. R., Wegener, D. T., MacCallum, R. C., \& Strahan, E. J. (1999). Evaluating the use of exploratory factor analysis in psychological research. Psychological Methods, 4, 272-299. doi:10.1037/1082-989X.4.3.272

Ginsburg, G.S. \& Silverman, W.K. (1995). Phobic anxiety disorders in Hispanic and Caucassian youth. Journal of Anxiety Disorders. 10(6), 517-528. doi:10.1016/S0887-6185(96)00027-8

Guarnieri, S., Ponti, L., \& Franca, T. (2010). The inventory of parent and peer attachment (IPPA): A study on the validity of styles of adolescent attachment to parents and peers in an Italian sample. TPM-Testing, Psychometrics, Methodology in Applied Psychology, 17(3), 103-130. Retrieved from http://search.proquest.com.ezproxy. fiu.edu/docview/821482981?accountid=10901

Hu, L., \& Bentler, P. M. (1999). Cutoff criteria for fit indexes in covariance structure analysis: Conventional criteria versus new alternatives. Structural Equation Modeling, 6, 1-55. doi:10.1080/10705519909540118

Ijzendoorn, M.H., Schuengel, C., \& Bakermans-Kraneburg, M.J. (1999). Disorganized attachment in early childhood: Meta-analysis of precursors, concomitants, and sequelae. Development and Psychopathology, 11, 225-249. doi:10.1017/S0954579499002035

Jaccard, J. \& Wan, C. K. (1996). LISREL approaches to interaction effects in multiple regression. Thousand Oaks, CA: Sage.

Johnson, L.N., Ketring, S.A., \& Abshire, C. (2003). The revised inventory of parent attachment: Measuring attachment in families. Contemporary Family Therapy, 25(3), 333-349. doi:10.1023/A:1024563422543

Kerns, K. A., Aspelmeier, J. E., Gentzler, A. L., \& Grabill, C. M. (2001). Parent-child attachment and monitoring in middle childhood. Journal of Family Psychology, 15, 69-81. doi:10.1037//0893-3200.15.1.69 
Kerns, K.A., Klepac, L., \& Cole, A.K. (1996). Peer relationships and preadolescence' perceptions of security in the child-mother relationship. Developmental Psychology, 32(3), 457-466. doi:10.1037/0012-1649.32.3.457

Kline, R. B. (1998). Principles and practice of structural equation modeling. New York, NY: Guilford Press.

Laible, D.J., Carlo, G., \& Raffaelli, M. (2000). The differential relations of parent and peer attachment to adolescent adjustment. Journal of Youth and Adolescence, 29(1), 45-59. doi:10.1023/A:1005169004882

Levy, K., Blatt, S., \& Shaver, P. (1998). Attachment styles and parental representations. Journal of Personality and Social Psychology, 74(2), 407-419. doi:10.1037/0022-3514.74.2.407

Lewis, M., Feiring, C., McGuffog, C., \& Jaskir, J. (1984). Predicting psychopathology in six-year-olds from early social relations. Child Development, 55, 123-136. Retrieved from http://www.jstor.org/stable/1129839

Main, M. \& Goldwyn, R. (1984). Adult attachment scoring and classification system. Unpublished manuscript, University of California at Berkley, Department of Psychology.

Main, M. \& Solomon, J. (1986). Discovery of a new, insecure-disorganized/disoriented attachment pattern. In T.B. Brazelton \& M. Yogman (Eds.), Affective development in infancy (pp. 95-124). Norwood: Ablex.

Marrone, M. (1998). Attachment and Interaction (pp. 238). London, England: Jessica Kingsley Publishers.

Marsh, H. W., Balla, J. R., \& McDonald, R. P. (1988). Goodness-of-fit indexes in confirmatory factor analysis: The effect of sample size. Psychological Bulletin, 103, 391-410. doi:10.1037/0033-2909.103.3.391

Minde, K., Minde, R., \& Vogel, W. (2006). Culturally sensitive assessment of attachment in children ages 18-40 months in a South African township. Infant Mental Health Journal, 27(6), 544-558. doi:10.1002/imhj.20106

Muris, P., Mayer, B., \& Meesters, C. (2000). Self-reported attachment style, anxiety, and depression in children. Social Behavior and Personality, 28, 157-162. Retrieved from http://search.proquest.com/docview/57726493?accountid=10901 
Muris, P., Meesters, C., Melick, M.V., \& Zwambag, L. (2001). Self-reported attachment style, attachment quality, and symptoms of anxiety and depression in young adolescents. Personality and Individual Differences, 30, 809-818. doi:10.1016/S0191-8869(00)00074-X

Muris, P., Meesters, C., Merckelbach, H., \& HuÈlsenbeck, P. (2000). Worry in children is related to perceived parental rearing and attachment. Behaviour Research and Therapy, 38, 487-497. doi:10.1016/S0005-7967(99)00072-8

Muris, P., Meesters, C., \& van den Berg, S. (2003). Internalizing and externalizing problems as correlates of self-reported attachment style and perceived parental rearing in normal adolescents. Journal of Child and Family Studies, 12 (2), 171183. doi: 10.1023/A:1022858715598

Muthén, L.K. and Muthén, B.O. (2007). Mplus User's Guide. Fifth Edition. Los Angeles, CA: Muthén \& Muthén.

Pace, C.S., San Martini, P., \& Zavattini, G.C. (2011). The factor structure of the Inventory of parent and peer attachment (IPPA): A survey of Italian adolescents. Personality and Individual Differences, 51, 83-88. doi:10.1016/j.paid.2011.03.006

Papini, D.R., Roggman, L.A., \& Anderson, J. (1991). Early-adolescent perceptions of attachment to mother and father: A test of the emotional-distancing and buffering hypothesis. Journal of Early Adolescence, 11(2), 258-275.

doi:10.1177/0272431691112006

Papini, D.R., \& Roggman, L.A. (1992). Adolescent perceived attachment to parents in relation to competence, depression, and anxiety: A longitudinal study. Journal of Early Adolescence, 12, 420-440. doi:10.1177/0272431692012004005

Parade, S.H., Leerkes, E.M., \& Blankson, A.N. (2010). Attachment to parents, social anxiety, and close relationships of female students over the transition to college. Journal of Youth and Adolescence, 39, 127-137. doi:10.1007/s10964-009-9396-x

Rosenstein, D.D. \& Horowitz, H.A. (1996). Adolescent attachment and psychopathology. Journal of Consulting and Clinical Psychology, 64(2), 244-253. doi: 10.1037/0022-006X.64.2.244

Rutter, M. (1995). Clinical implications of attachment concepts: Retrospect and prospect. Journal of Child Psychology and Psychiatry, 36(4), 549.

doi: 10.1111/j.1469-7610.1995.tb01311.x

Shmueli-Goetz, Y., Target, M., Fonagy, P. \& Datta, A. (2008). The child attachment interview: A psychometric study of reliability and discriminant validity. Developmental Psychology, 44(4), 939-956. doi: 10.1037/0012-1649.44.4.939 
Silverman, W. K., \& Albano, A. M. (1996). Anxiety Disorders Interview Schedule for Children for DSM-IV: (Child and Parent Versions). San Antonio, TX:

Psychological Corporation.

Suomi, S.J. (1999). Attachment in rhesus monkeys. In J. Cassidy \& P.R. Shaver (Eds.) Handbook of attachment: Theory, research, and clinical implications. New York: Guilford Press.

Sue, S. (1999). Science, ethnicity, and bias: Where have we gone wrong? American Psychologist, 54(12), 1070, 1077. doi:10.1037/0003-066X.54.12.1070

Tabachnick, B. G., \& Fidell, L. S. (2001). Using multivariate statistics (4th ed.). Boston: Allyn \& Bacon.

United States Department of Health and Human Services. (2001). Mental health: Culture, race, and ethnicity - a supplement to mental health: A report of the Surgeon General. Rockville, MD: U.S. Department of Health and Human Services, Public Health Service, Office of the Surgeon General.

United States Census Bureau. (2000). Census 2000 Redistricting Data (Public Law 94-171). Retrieved February 15, 2011, Retrieved from http://www.census.gov/population/www/socdemo/hispanic/hispanic.html

Vignoli, E. \& Mallet, P. (2004). Validation of a brief measure of adolescents' parent attachment based on Armsden and Greenberg's three-dimension model. Revue Européenne de Psychologie Appliquée, 54, 251-260. doi:10.1016/j.erap.2004.04.003

Warren, S.L., Huston, L., Egeland, B., \& Sroufe, A.L. (1997). Child and adolescent anxiety disorders and early attachment. Journal of the American Academy of Child \& Adolescent Psychiartry, 35(5), 637-644. doi:10.1097/00004583-199705000-00014

Wothke, W. (2000). Longitudinal and Multigroup modeling with missing data. In T. D. Little, K. U. Schnabel, and J. Baumert (Eds.), Modeling longitudinal and multilevel data: practical issues, applied approaches, and specific examples. (pp. 197-216). Mahwah, NJ: Lawrence Erlbaum. 
APPENDICIES 
Appendix 1- Frequency and Percentages of Primary Diagnoses 
Table 1

Frequency and Percentages of Primary Diagnoses

\begin{tabular}{lrr}
\hline Primary Diagnoses & & \\
$(n=282)$ & $n$ & $\%$ \\
\hline Separation Anxiety & 97 & 34.4 \\
Social Phobia & 55 & 19.5 \\
Specific Phobia & 32 & 11.3 \\
Panic Disorder & 1 & .4 \\
Panic Disorder with Agoraphobia & 5 & 1.8 \\
Agoraphobia without Panic Disorder & 1 & .4 \\
Generalized Anxiety Disorder & 30 & 10.6 \\
Obsessive Compulsive Disorder & 6 & 2.1 \\
Dysthymia & 2 & .7 \\
Major Depression & 5 & 1.8 \\
Attention Deficit Hyperactivity Disorder & 6 & 2.1 \\
(Combined Type) & & \\
Oppositional Defiant Disorder & 6 & 2.1 \\
Selective Mutism & 6 & 2.1 \\
No DX & 13 & 4.6 \\
Not available & 15 & 5.4 \\
\hline
\end{tabular}


Appendix 2 - Factor loadings of IPPA-M items 
Table 2

Factor loadings of IPPA-M items

\begin{tabular}{|c|c|c|}
\hline IPPA-M Items & $\begin{array}{l}\text { Factor } 1 \\
\text { (factor } \\
\text { loadings) }\end{array}$ & $\begin{array}{l}\text { Factor } 2 \\
\text { (factor } \\
\text { loadings) }\end{array}$ \\
\hline My mother understands me & .783 & \\
\hline $\begin{array}{l}\text { When I am angry about something, my mother tries to be } \\
\text { understanding }\end{array}$ & .753 & \\
\hline $\begin{array}{l}\text { I can count on my mother when I need to get something off my } \\
\text { chest }\end{array}$ & .750 & \\
\hline I tell my mother about my problems and troubles & .689 & \\
\hline My mother helps me to talk about my differences & .678 & \\
\hline I trust my mother & .677 & \\
\hline My mother respects my feelings & 649 & \\
\hline My mother does a good job as my mother & 639 & \\
\hline $\begin{array}{l}\text { When we discuss things, my mother cares about my point of } \\
\text { view }\end{array}$ & .638 & \\
\hline $\begin{array}{l}\text { If my mother knows something is bothering me, she asks me } \\
\text { about it }\end{array}$ & .592 & \\
\hline My mother helps me to understand myself better & .570 & \\
\hline My mother trusts my judgment & .561 & \\
\hline $\begin{array}{l}\text { I like to get my mothers point of view on things I'm concerned } \\
\text { about }\end{array}$ & .520 & \\
\hline My mother can tell when I'm upset about something & .519 & \\
\hline My mother accepts me as I am & .425 & \\
\hline I get upset easily around my mother & & .676 \\
\hline I get upset a lot more than my mother knows about. & & .648 \\
\hline $\begin{array}{l}\text { Talking over my problems with my mother makes me feel } \\
\text { ashamed or foolish }\end{array}$ & & .617 \\
\hline My mother expects too much from me & & .585 \\
\hline I feel it's no use letting my feelings show around my mother & & .569 \\
\hline I don't get much attention from my mother & & .485 \\
\hline My mother doesn't understand what I'm going through these days. & & .470 \\
\hline I feel angry with my mother & & .466 \\
\hline My mother has her own problems, so I don't bother her with mine. & & .463 \\
\hline I wish I had a different mother & $\mathrm{N} / \mathrm{A}$ & $\mathrm{N} / \mathrm{A}$ \\
\hline
\end{tabular}


Appendix 3 - Scree Plot generated by Exploratory Factor Analysis 
Figure 1

Scree plot generated the EFA

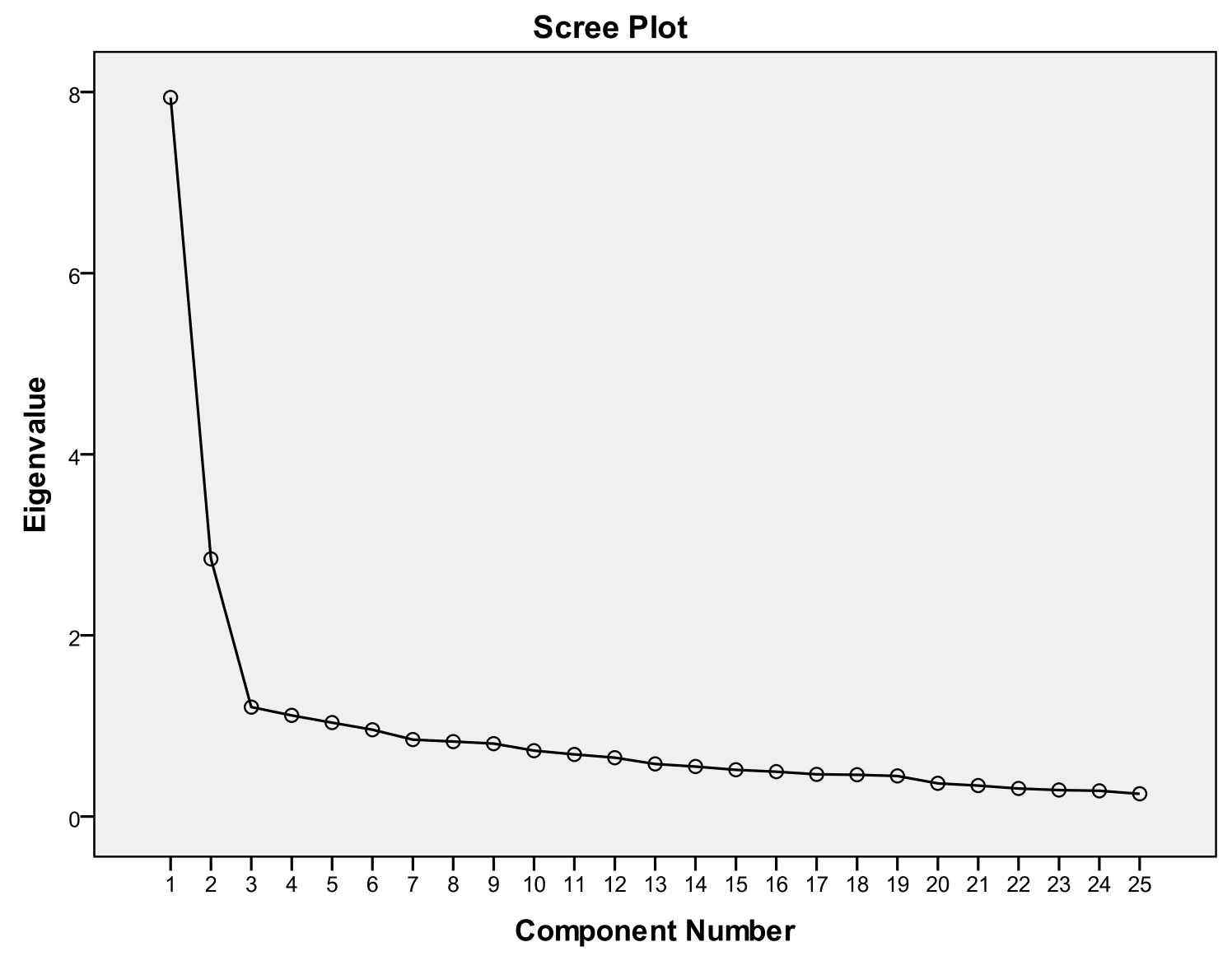

